\title{
La plancha de plomo en cubiertas $y$ fachadas
}

ROCIO ARAMBURU MAQUA

Leda. en Ciencias Físicas

Centro de Información de la Asociación Nacional del Plomo

\section{$R E S U M E N$}

Se describen las caracteristicas generales de la plancha de plomo con vistas a su utilización en cubiertas y fachadas. Se resumen las reglas básicas de instalación de la plancha en cubiertas y fachadas, en particular las que se refieren al tamaño de las piezas, características de las juntas y elementos de fijación, $y$ condiciones de estanquidad. Se indican

la compatibilidad del plomo con otros materiales de construcción para su instalación, así como las normas españolas de interés en este campo.

\section{$S U M M A R Y$}

$A$ description is given of the properties of lead sheet for roofing and cladding purposes. Basic rules as far as installations are concerned are given, specially with respect to dimensions of the pieces, characteristics of joints and fixings, and watertightness conditions. Compatibility of lead with other building materials is also taken into account, as well as elementary health and safety rules for its installation. Spanish standards on the subject are also reviewed.

El empleo de la plancha de plomo en cubiertas se remonta a muchos años atrás, como lo atestiguan un gran número de edificios históricos, entre los que se encuentran el Palacio Real de Madrid (fig. 1), Notre Dame de Paris y el Palacio de Versalles.

Actualmente se está extendiendo su utilización en toda Europa gracias a la riqueza de posibilidades que ofrece su empleo en la construcción, debidas a sus propiedades fisicas y químicas. Estas hacen del plomo un metal duradero, fácil de manejar, resistente a la corrosión atmosférica y que armoniza perfectamente con los demás materiales de construcción.

Los paises del Mercado Común utilizan anualmente más de 125.000 toneladas de plancha y lámina de plomo, de las que una fracción apreciable se destina a la restauración de edificios históricos (fig. 2). Sin embargo, en la actualidad la aplicación más importante de la plancha de plomo en el exterior de los edificios es como accesorios de cubiertas (limas, baberos, etc...), ya sea en edificios nuevos o en obras de rehabilitación.

Recientemente se han encontrado nuevas aplicaciones de la plancha de plomo para el revestimiento de exteriores (fig. 3), a la vez que ha habido un resurgir de su empleo tradicional para el recubrimiento de tejados (figs. 4 y 5).

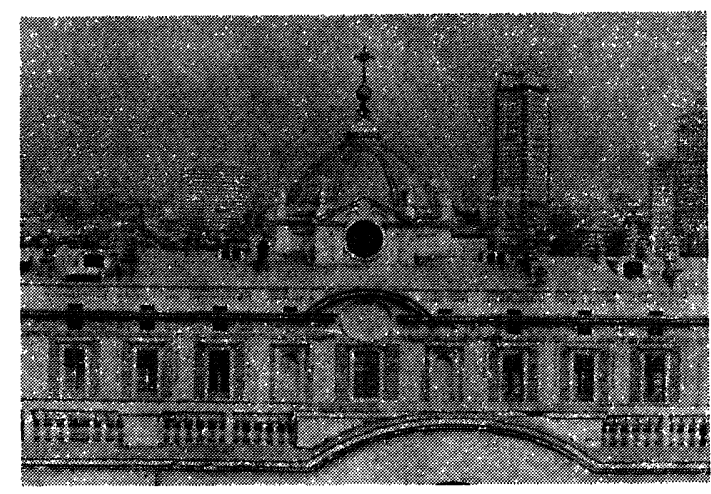

Fig. 1.-Palacio Real de Madrid. Cubierta del ala Norte y de la cúpula de la Capilla Real. Fotografia tomada con autorización del Patrimonio Nacional y con la colaboración del Centro Nacional de Investigaciones Metalúrgicas. 


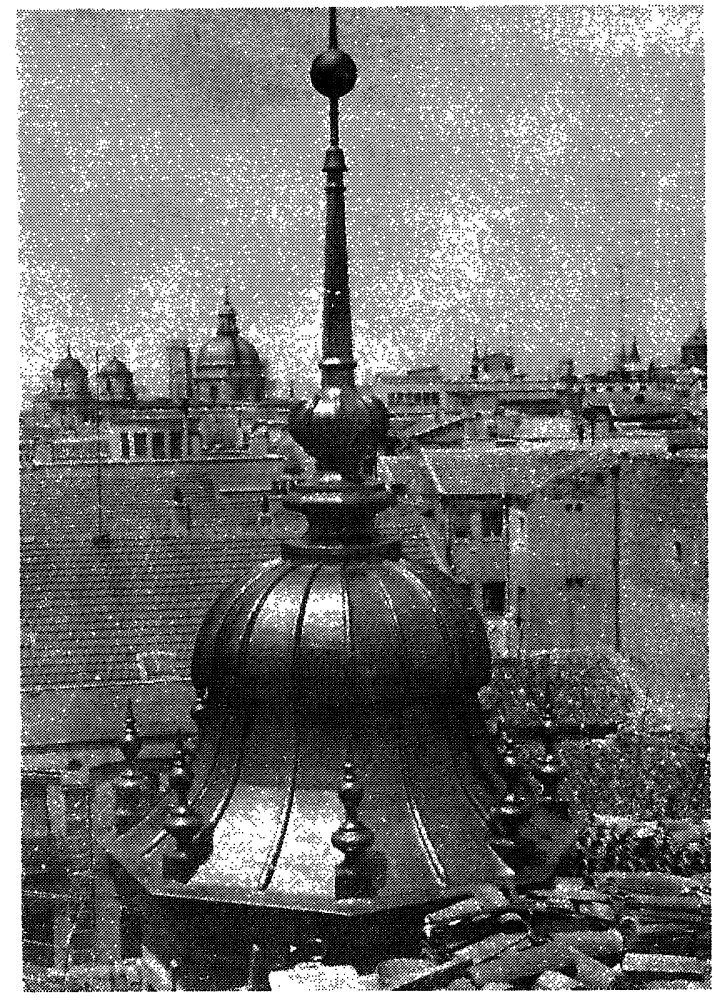

Fig. 2.-Iglesia de San Cayetano en Madrid. Cúpula restaurada. Foto cedida por la Empresa MARTOS.

Fig. 5.-Edificio de oficinas en Edimburgo.

Foto cedida por la Lead Development Association.

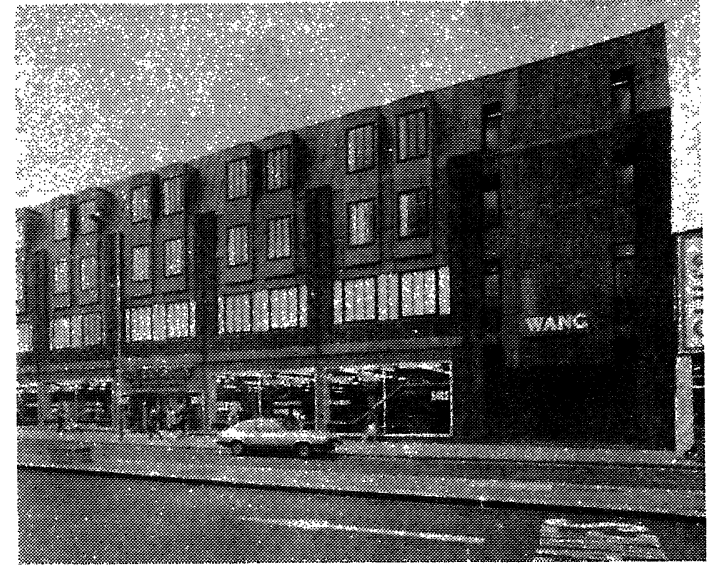

Fig. 3.-Edificio de oficinas y tiendas en Teddington. Foto cedida por la Lead Development Association.

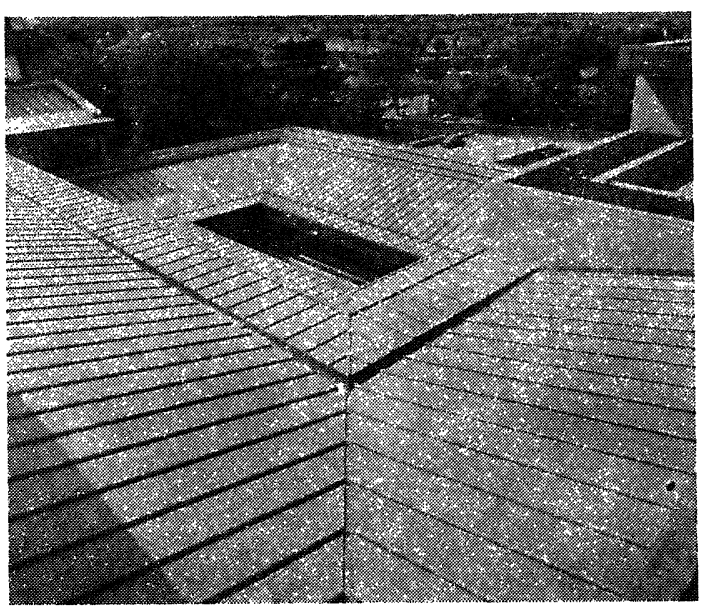

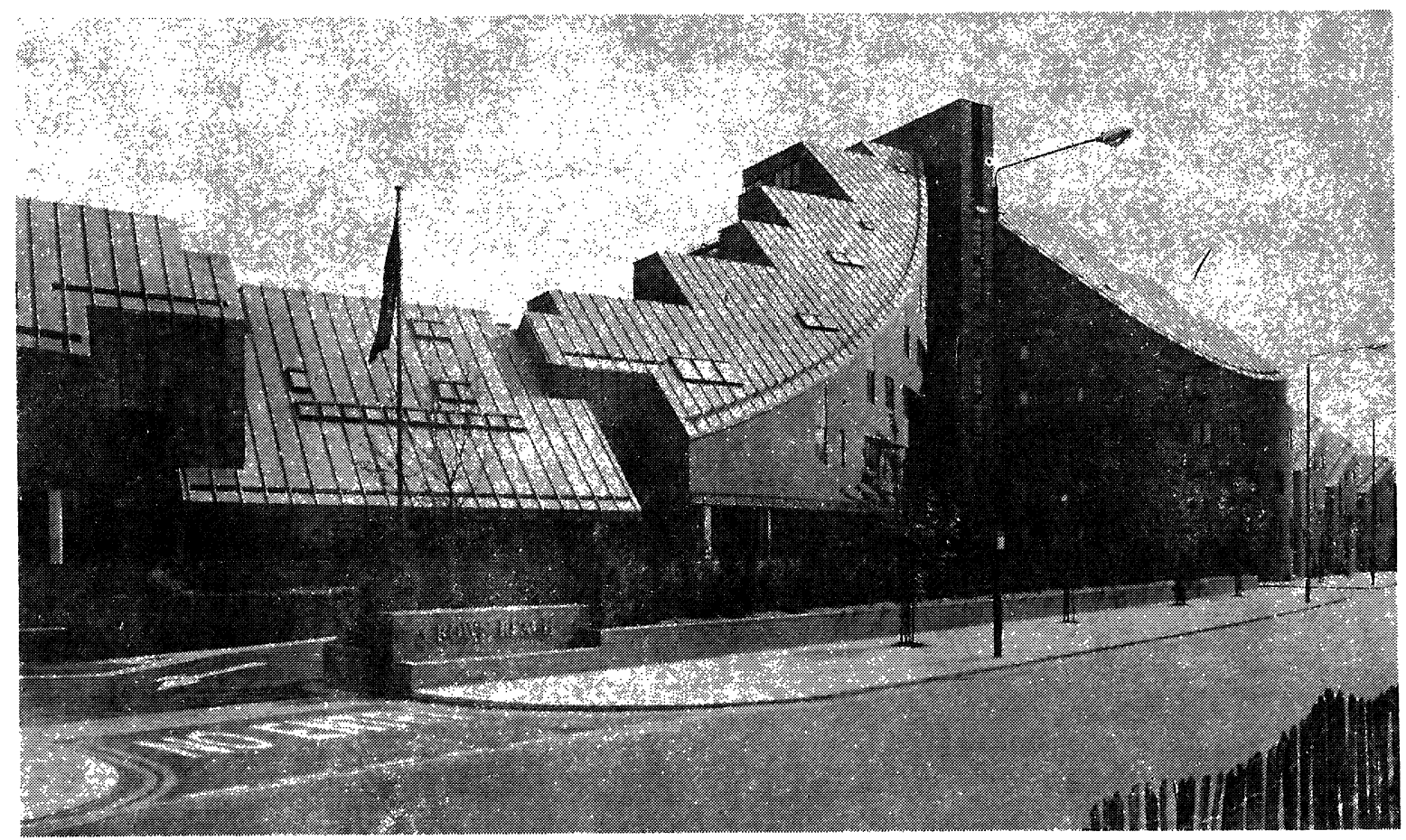

Fig. 4.-Bloque de apartamentos en Chelsea, Londres. Foto cedida por la Lead Development Association. 


\section{CARACTERISTICAS DE LA PLANCHA DE PLOMO}

El plomo, gracias a sus características mecánicas y propiedades fisicas y quimicas, tiene una gran variedad de aplicaciones en la construcción, especialmente en edificios.

En cubiertas, la plancha de plomo ofrece un conjunto de ventajas que ningún otro material puede proporcionar.

- La plancha de plomo se instala fácilmente, es soldable, fácil de cortar, y se puede trabajar con herramientas manuales sencillas, sin riesgo de rotura.

- La larga duración del plomo reduce al mínimo la reparación de las cubiertas.

- La maleabilidad de las planchas de plomo, empleadas como accesorios de cubiertas, facilita el mantenimiento de los tejados, simplemente levantando la plancha para efectuar las reparaciones convenientes.

- El plomo es un material económico a causa de su duracion, calidad y fácil mantenimiento. Otro elemento que interviene en su favor es que conserva su valor como metal, y se puede vender a buen precio después de largos años de utilización.

- La maleabilidad de la plancha de plomo hace que se adapte perfectamente a la superficie sobre la que se coloca aunque ésta tenga irregularidades, consiguiendo así una excelente estanquidad.

- La elevada densidad del plomo, 11,35, lo coloca a la cabeza de todos los materiales para aislar acústicamente de ruidos aéreos (granizo, lluvia, tráfico aéreo ...). Además, este metal tan pesado proporciona una gran estabilidad a las cubiertas, haciéndolas resistentes a los vientos e incluso tempestades, siendo de gran utilidad al borde del mar y en la cumbre de las montañas.

- Otra propiedad importante del plomo a tener en cuenta es su resistencia al fuego. Las planchas de plomo son incombustibles y aseguran una protección adecuada contra la propagación del fuego en el interior de un edificio o de un edificio a otro, tanto en cubiertas como en recubrimientos de fachadas.

- La plancha de plomo en las cubiertas proporciona seguridad de acceso con riesgo mímino de resbalar accidentalmente.

- El plomo es uno de los metales puros que tiene mejor resistencia a la corrosión atmosférica, ya sea urbana, rural o marina. Recién cortado, el plomo expuesto a la intemperie se recubre de una fina capa de óxido sobre la que no tarda en aparecer, por reacción con el anhídrido carbónico de la atmósfera, una pátina de carbonato de plomo. La penetracion de esta fina capa protectora en el metal es insignificante; en efecto, medidas realizadas al cabo de diez años de exposición en diferentes atmósferas indican una penetración media de $0,06 \mathrm{~mm}$, cuya velocidad disminuye rápidamente con el tiempo. La pátina expuesta a humos industriales puede contener sulfato, además de carbonato, pero sigue manteniendo su condición protectora. El color natural de la pátina del plomo es gris-plata, que a veces se torna más oscuro debido al tizne que se posa en su superficie. Dado que la pátina es insoluble al agua de lluvia no arrastra nada en solución, con lo que no mancha ni deteriora los materiales adyacentes. 


\section{INSTALACION DE LA PLANCHA DE PLOMO}

\section{Reglas básicas de instalación}

El plomo tiene una resistencia mecánica moderada y un coeficiente de dilatación lineal relativamente elevado (alrededor de 2,5 veces el del hierro) que hace posible fenómenos de deformación por fluencia si no se toman precauciones elementales en su colocación. Resulta evidente que si se realizan cubiertas en superficies continuas (en un solo bloque o soldadas), con los cambios de temperatura tendrán lugar dilataciones y contracciones que al no poder producirse libremente, ocasionarán fisuras a largo plazo, perdiendo la cubierta su propiedad más importante: la estanquidad.

Por lo tanto es importante limitar las dimensiones de las planchas de plomo, de modo que la cantidad de movimiento térmico no sea excesiva, y asegurar libertad para este movimiento. Esto se consigue mediante la aplicación de tres principios básicos:

\section{Se debe limitar el tamaño de las piezas}

Las piezas deben ser más pequeñas cuanto menor sea su espesor, de modo que la dilatación y contracción naturales sean mínimas, evitando por tanto el riesgo de distorsiones importantes y el consiguiente peligro de agrietamiento por fatiga.

En la tabla adjunta se dan los valores límites de longitud y anchura en función del espesor de la plancha.

\begin{tabular}{|c|c|c|}
\hline $\begin{array}{c}\text { Espesor } \\
(\mathbf{m m})\end{array}$ & $\begin{array}{c}\text { Anchura } \\
(\mathbf{m m})\end{array}$ & $\begin{array}{c}\text { Longitud } \\
\mathbf{( m m )}\end{array}$ \\
\hline 2,0 & 600 & 1.500 \\
2,5 & 650 & 2.000 \\
3,0 & 675 & 2.300 \\
\hline
\end{tabular}

\section{Las juntas y los elementos de fijación deben permitir las dilataciones}

Las juntas y las fijaciones deben estar concebidas de modo que permitan las variaciones dimensionales debidas a los cambios de temperatura sin provocar deformaciones importantes, ni riesgo de rotura por fatiga. Hay que tener en cuenta que las variaciones totales de temperatura en una cubierta pueden ser de hasta $100^{\circ} \mathrm{C}$, lo que supone una variación de unos $3 \mathrm{~mm}$ por metro. Es esencial que las fijaciones de las planchas de plomo sobre su base se efectúen en las juntas.

Es indispensable emplear clavos de cobre para evitar la corrosión.

\section{Las juntas deben garantizar la estanquidad}

La realización de las uniones debe permitir una estanquidad absoluta al agua en todas las condiciones atmosféricas posibles. En efecto, toda filtración, incluso excepcional, puede provocar un deterioro de la base que puede perjudicar la calidad de la cubierta. Para asegurar la estanquidad, es preciso que exista un desnivel superior a la altura máxima de agua previsible en el caso de que las dos planchas se unan por una simple superposición, o bien que se realice un cierre estanco entre láminas adyacentes mediante un engatillado adecuado.

El plomo colocado correctamente permite realizar revestimientos perfectamente estancos.

Hoy dia es preciso cumplir con más rigor que en el pasado esta serie de reglas básicas, debido a que para muchas aplicaciones se están especificando espesores menores de los empleados en épocas más prósperas. 


\section{Condiciones de la base}

Cualquier material de construcción sirve como sustrato para recubrir con planchas de plomo, siempre que ofrezca una superficie lisa continua, sea lo suficientemente consistente para sostener los puntos de fijación permanente, y aguantar el peso del plomo. Es importante que el material de base sea duradero, ya que el recubrimiento también lo es.

Conviene emplear un material aislante entre el plomo y su base; generalmente se utiliza papel parafinado o un revestimiento bituminoso de buena calidad que no se reblandezca con el calor.

\section{Compatibilidad del plomo con otros materiales}

\section{a) Cemento}

Ciertos cementos de tipo "Portland" pueden desprender, al colocarlos, productos básicos, causa esencial de una corrosión lenta del plomo en presencia de humedad; por tanto es recomendable interponer un aislante entre el plomo y el cemento.

\section{b) Madera}

Se debe evitar el contacto directo del plomo con ciertas maderas duras (roble, castaño, teca ...), particularmente en medio húmedo, a causa del ácido fórmico y acético que contienen estas maderas; por lo tanto es necesario interponer un aislante entre el plomo y la madera, facilitar la ventilación debajo del plomo y utilizar preferentemente maderas blandas (pino) que actúan poco o nada sobre el plomo.

\section{c) Yeso}

En principio el yeso no ataca al plomo, sin embargo, se aconseja interponer un aislante, particularmente cuando el yeso está todavia fresco.

\section{d) Otros metales}

El plomo, en contacto con otros metales y en medio húmedo, es raramente atacado por efecto del par galvánico. De todos modos se debe evitar el contacto directo del plomo con otros metales menos nobles, sobre todo si éstos son en forma de piezas relativamente pequeñas en relación con las de plomo. La diferencia de potencial electroquimico podria, en este caso, favorecer un ataque más rápido de metales como el aluminio, zinc y acero.

\section{Calidad}

El plomo empleado habitualmente en España es el doblemente refinado, con un máximo de impurezas del 0,03\% en peso (UNE 37-201-77 "Plomo. Definiciones y calidades"). Sin embargo, ensayos llevados a cabo recientemente han mostrado que el plomo-cobre $(0,02$ a $0,04 \%$ de cobre) tiene una resistencia a la fatiga superior.

\section{Medidas}

Las medidas y tolerancias de las planchas y láminas de plomo están expresadas en la norma UNE 37-203-74 "Planchas y láminas de plomo. Definiciones, calidades, medidas y tolerancias". 
Los espesores corrientemente empleados en cubiertas y recubrimientos son de 2, 2,5 y $3 \mathrm{~mm}$. Las tolerancias de espesor están expresadas por las tolerancias sobre la masa de la plancha, según la tabla adjunta.

\begin{tabular}{|l|c|c|}
\hline $\begin{array}{c}\text { Espesor } \\
\mathbf{~ m m}\end{array}$ & $\begin{array}{c}\text { Masa por } \mathbf{m}^{2} \\
\mathbf{k g}(\mathbf{1})\end{array}$ & $\begin{array}{c}\text { Tolerancia } \\
( \pm \mathbf{1 0} \mathbf{\%}) \mathbf{k g}\end{array}$ \\
\hline 2 & 22,7 & 2,27 \\
2,5 & 28,4 & 2,84 \\
3 & 34,1 & 3,41 \\
\hline
\end{tabular}

(1) Densidad $11,35 \mathrm{~kg} / \mathrm{dm}^{3}$.

Las planchas y láminas de plomo se suministran en ancho normal de 1 metro, y en longitud normal de 5 metros, o cortadas a las medidas solicitadas.

Las bandas de plomo, con un ancho inferior a $500 \mathrm{~mm}$, según se indica en la misma norma, se suministran en los anchos de 100,120,140,160, 250, 300 y $500 \mathrm{~mm}$.

\section{Reglas de higiene y seguridad}

La manipulación de las planchas de plomo no comporta ningún riesgo, a condición de respetar algunas reglas elementales de higiene.

El plomo es tóxico sólo en el caso de absorción repetida, ya sea en forma de metal o de compuesto por via digestiva o respiratoria. Por lo tanto es necesario:

- lavarse las manos antes de comer o fumar, en caso de manipulación continua de plomo;

- prever una aireación suficiente cuando se ejecuta una soldadura autógena de plomo en un local cerrado.

Es decir, que las precauciones a tomar en la instalación de la plancha de plomo son las mismas que dictaria el sentido común para la instalación de cualquier otro material de construcción. 